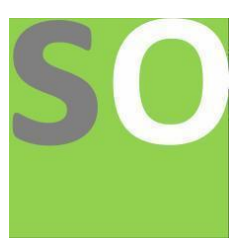

Article title: Acute inflammatory Transverse Myelitis post Pfizer-BioNTech-COVID-19 vaccine in 16-year-old Authors: Ammar Albokhari[1], Abdulrhman Alsawas[2], Mohammad Adnan[3], Abdulaziz Alasmari[4], Sarah Aljuhani[5], Mousa Almejalli[6], Hanaa Kedah[7]

Affiliations: Internal Medicine, Hera General Hospital, Makkah, Saudi Arabia[1], Public Health, Hera General Hospital, Makkah, Saudi Arabia[2]

Orcid ids: 0000-0003-0112-7997[1]

Contact e-mail: ammarxv@gmail.com

License information: This work has been published open access under Creative Commons Attribution License http://creativecommons.org/licenses/by/4.0/, which permits unrestricted use, distribution, and reproduction in any medium, provided the original work is properly cited. Conditions, terms of use and publishing policy can be found at https://www.scienceopen.com/.

Preprint statement: This article is a preprint and has not been peer-reviewed, under consideration and submitted to ScienceOpen Preprints for open peer review.

DOI: 10.14293/S2199-1006.1.SOR-.PPVXII5.v1

Preprint first posted online: 11 October 2021

Keywords: Transverse myelitis, Pfizer, Saudi Arabia, Teenager, complication, BioNTech, COVID-19, vaccination, Corona, COVID-19 vaccine 


\title{
Acute inflammatory Transverse Myelitis post Pfizer-BioNTech-COVID-19 vaccine in 16-year-old
}

Ammar Albokhari, Abdulrhman Alsawas, Mohammad Adnan, Abdulaziz Alasmari, Sarah Aljuhani, Mousa Almejalli, and Hanaa Kedah.

\begin{abstract}
Coronavirus disease 2019 (COVID-19) originated in China in early March 2019. Saudi Food and Drug Authority approved the registration of the Pfizer-BioNTech COVID-19 vaccine in Saudi Arabia on December 10, 2020 and on May 10, 2021, Pfizer-BioNTech was given an authorized emergency use in 12 to 15 years old children. Saudi Arabia's Ministry of Health started Pfizer-BioNTech COVID-19 vaccination for 12 to 18 years old on June 27,2021 . Here we have a case of 16-year-old female admitted to the medical ward diagnosed with acute inflammatory transverse myelitis after two weeks from second dose of the Pfizer-BioNTech COVID-19 vaccine. The diagnosis was based on normal laboratory workup but significant radiological findings. She was discharged after a full recovery. There are multiple cases of post-vaccine acute inflammatory transverse myelitis shared by medical journals, but due to lack of literature review for the teenager population, we think our case may be the first case of acute inflammatory transverse myelitis following second dose of Pfizer-BioNTech COVID-19 vaccine in this population.
\end{abstract}

Keywords: Transverse myelitis, Pfizer, Saudi Arabia, Teenager, complication

\section{Introduction}

Coronavirus disease 2019 (COVID-19) has claimed deaths of many people since its outbreak and declaration by WHO as a pandemic. Several research institutions and companies have made substantial efforts to develop COVID-19 vaccines [1]. Upon approval by the regulatory agencies Medicines and Healthcare Products Regulatory Agency (MHPRA) and Food and Drug Administration (FDA), and WHO, COVID-19 vaccines have been used globally to suppress the virus. Various COVID-19 vaccines available in the market include Pfizer-BioNTech, AstraZeneca, Moderna, Johnson \& Johnson, and Sinovac vaccines $[2,3]$. Cases of vaccine adverse effects reported the FDA Adverse Event Reporting System (FAERS) as of March 2, 2021, include neurological symptoms such as paresthesia, muscle spasm, headache, myalgia, pain, and dizziness [4]. Rare cases of transverse myelitis, stroke, acute disseminated encephalomyelitis, facial palsy, tinnitus, tremor, seizure, dysphonia, and diplopia have also been reported in the literature.

Transverse myelitis is a medical condition emanating from spinal cord inflammation, which is a characteristic of neurological dysfunction of the sensory and motor tracts located on both sides of the spinal cord. Studies report transverse myelitis as a complication due to COVID-19 infection. Several 
case reports have linked neurological complications such as transverse myelitis to severe acute respiratory syndrome coronavirus 2 (SARS-CoV-2) infection [5-11]. The clinical cases involved both males and females, aged between 28 and 63 years. The tests conducted initially confirmed that the patients were SARS-CoV-2 positive. Blood analysis indicated leukopenia in addition to a slightly higher level of C-reactive protein (CRP). Spine MRI showed hyperdensity, especially in the transverse region. The lumbar puncture results and blood neutropenia conclusively suggest viral pathogen as the causative agent [7].

Similarly, several transverse myelitis cases and other complications arising from COVID-19 vaccination, including thrombosis with thrombocytopenia syndrome (TTS), have been reported on CDC and FDA Vaccine Adverse Event Reporting System (VAERS) [12-16]. The FDA VAERS system has reported 45 cases of transverse myelitis as of 27 April 2021. Five of the cases occurred after the Janssen vaccine, 19 after the Pfizer-BioNTech vaccine while 21 cases occurred after the Moderna vaccine.

\section{Case Presentation}

A 16-year-old female, otherwise healthy, presented to the emergency department of the hospital with lower extremity weakness and difficulty in walking for three days. Detailed medical history revealed that the patient was administered a second dose of Pfizer-BioNTech-COVID-19 vaccine on July 26,2021 , and two days after the administration, the patient noticed weakness in her lower extremity. As the days went by the weakness gradually progressed to her upper extremity with numbness of both the lower limbs; and finally on August 11, 2021, the patient sought medical care. There was no history of being febrile before vaccination; no bowel or bladder dysfunction, headache, seizure, or loss of consciousness was reported. A physical examination was conducted to show that her temperature was 37 degrees Celsius, her blood pressure was 110/65 $\mathrm{mmHg}$ with a heart rate of 85 beats per minute, and $100 \%$ oxygen saturation with a respiratory rate of 18 breaths per minute. The neurological examination was unremarkable except for a moderate decline in the power of all extremities, decrease sensation to fine and pain stimuli in the lower extremity, increased tone with spasticity pattern, and hyperreflexia with present Babinski sign. Cranial nerves and cerebellum examination were unremarkable.

Haematology tests gave normal results with a complete blood count. Biochemistry tests show normal results (Table 1) and Brucellosis titer was negative.

\begin{tabular}{|l|c|c|l|}
\hline Test & Patient Value & Normal Range & Unit \\
\hline WBC & 8.31 & $4-10$ & $10 * 3 / \mathrm{uL}$ \\
\hline HGB & 12.8 & $12-15$ & $\mathrm{~g} / \mathrm{dL}$ \\
\hline HCT & 36.5 & $40-50$ & $\%$ \\
\hline
\end{tabular}




\begin{tabular}{|l|c|c|l|}
\hline MCV & 78.8 & $83-101$ & $\mathrm{fL}$ \\
\hline MCH & 27.6 & $27-32$ & $\mathrm{pg}$ \\
\hline PLT & 285 & $150-400$ & $10 * 3 / \mathrm{ul}$ \\
\hline PT & 12.9 & $11.5-15.5$ & $\mathrm{sec}$ \\
\hline PTT & 36.7 & $26-40$ & $\mathrm{sec}$ \\
\hline INR & 1.170 & $0.9-1.2$ & $\mathrm{INR}$ \\
\hline ALT & 7.8 & $16-63$ & $\mathrm{U} / \mathrm{L}$ \\
\hline AST & 13.7 & $15-37$ & $\mathrm{U} / \mathrm{L}$ \\
\hline NA & 138 & $136-145$ & $\mathrm{Mmol} / \mathrm{L}$ \\
\hline K & 3.93 & $35-5.1$ & $\mathrm{Mmol} / \mathrm{L}$ \\
\hline BUN & 2.2 & $2-6$ & $\mathrm{Mmol} / \mathrm{L}$ \\
\hline Creatinine & 36 & $44-80$ & $\mathrm{Umol} / \mathrm{L}$ \\
\hline ESR & 8 & $0-20$ & $\mathrm{Mm} / \mathrm{hr}$. \\
\hline CRP & 0.0156 & $<0.3 \mathrm{mg} / \mathrm{dl}$ & $\mathrm{Mg} / \mathrm{dl}$ \\
\hline Table 1 & & & \\
\hline
\end{tabular}

Results for the magnetic resonance imaging (MRI) implied an acute inflammation on the spine as shown by the dorsal spinal cord, including contrast of the cervical (Figure 1), and thoracic (Figure 2) spinal cord, with normal MRI of the brain (Figure 3).

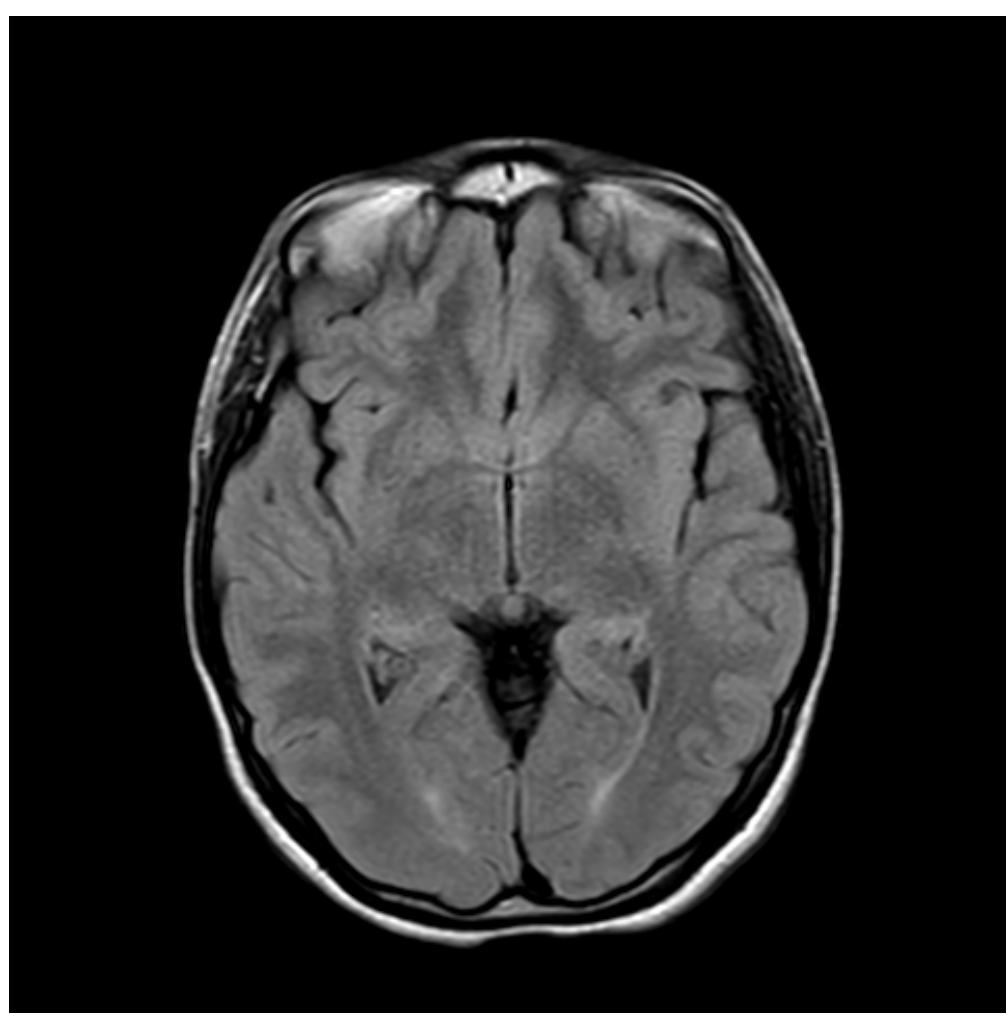



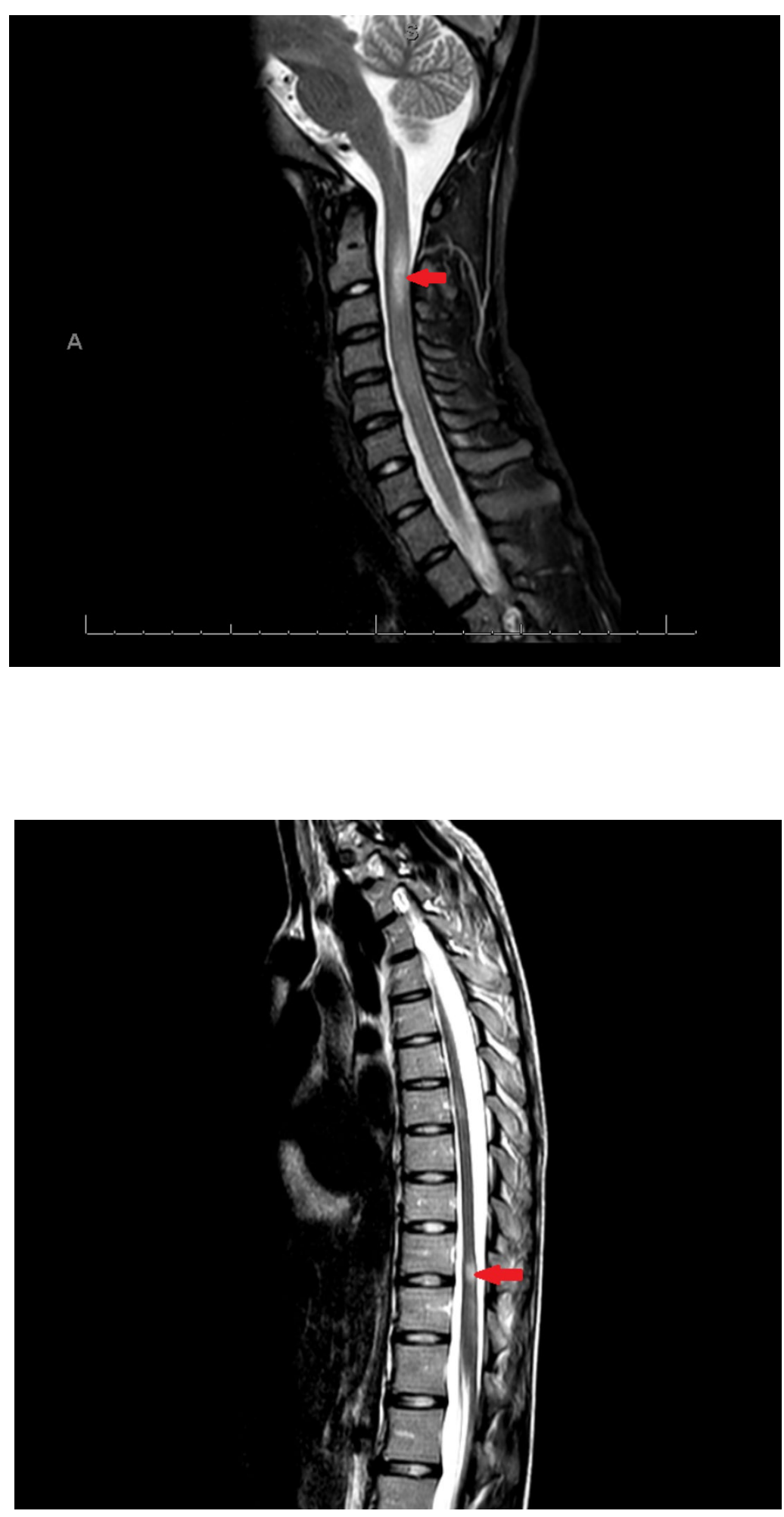


\section{Discussion}

Transverse myelitis is a disorder characterized by the spinal cord focal inflammation that usually does not proceed to a painless chronic lesion. Major known causes of transverse myelitis are infectious like viral, bacterial or fungal infections and post infectious immune triggered disorder like Acute Disseminated Encephalomyelitis (ADEM) and Multiple Sclerosis (MS) and most of the above disorders have been ruled out by presence of normal laboratory findings and normal brain MRI in our patient; one of the known causes of immune triggered transverse myelitis is post vaccine including COVID-19 which we reporting in our case [17]. Viruses that are commonly linked to transverse myelitis are the herpes viruses particularly the zoster virus that causes chickenpox and the one that causes shingles. Most cases of transverse myelitis occur after recovery from an infection. Other rare causes of transverse myelitis are systemic autoimmune diseases; for instance, systemic lupus erythematosus Sjögren's syndrome that injures the spinal cord. Although many cases of transverse myelitis are correctly diagnosed, the actual causes for $30 \%$ of the cases are not known [18].

Vaccines have also been reported as a potential cause of transverse myelitis $[19,20]$. Other case reports involving transverse myelitis due to administration of COVID-19 vaccines exhibit almost similar symptoms and results [21-25]. Malhotra et al. reported their first case of transverse myelitis as an adverse event following the administration of AstraZeneca/Oxford (viral-vectored, recombinant ChAdOX1) COVID-19 vaccine [24]. The patient who registered abnormal sensations on the lower limbs received the AZD1222 (ChAdOx1) vaccine eight days ago. Results for the MRI of the spine revealed "an ovoid T2-hyperintense lesion in the dorsal aspect of the spinal cord at C6 and C7 vertebral levels" which signaled demyelination as a result of vaccination [24].

Fitzsimmons and Nance also reported a case of transverse myelitis due to the Moderna vaccine [25]. The patient reported increasing lower back pain along with pain and numbness in the calves of both legs, which extended to the ankle. Laboratory tests and MRI of the lumbar and cervical regions of the spine revealed an increased $\mathrm{T} 2$ cord signal in the distal spinal cord and conus, suggestive of transverse myelitis due to vaccination [25]. 
The majority of the transverse myelitis cases following COVID-19 vaccination were treated with $1 \mathrm{~g}$ of intravenous methylprednisolone for five days of which full recovery was reported in almost all the cases. Among the 133,321 reported cases by VAERS as of April 27, 2021, only 45 (0.003\%) cases of transverse myelitis were reported. This is a confirmation that transverse myelitis is a very rare disorder. This is further confirmed by the statistics presented by Baxter et al. whose systemic review found only seven cases of transverse myelitis after administering approximately 64 million doses of vaccine [26].

Study limitation: Lumbar puncture (LP), for finding cerebral spinal fluid (CSF) protein level, was not done as brain and spinal cord MRI study was sufficient for diagnosis and the patient showed rapid improvement with the treatment given.

\section{Conclusion}

Our 16-year-old patient was diagnosed with transverse myelitis that appeared after two days of administering the second dose of Pfizer-BioNTech COVID-19 vaccine and required hospitalization and management for five days. We publish our case for health administration to take notice of the possibility of serious adverse effects in the teenager population.

\section{Additional Information:}

Ethic approval and consent to participate: Institutional Review Board-Makkah approved research conduct at Sep/10/2021 with reference number

Human Subject: consent was obtained verbally and written from the parent of the patient - as the patient is underage of 18-years-old -.

Funding: no funding was needed/received.

Availability of data and materials: the submitting primary author has the research data.

Conflict of interest: with the completed form of International Committee of Medical Journal Editors (ICMJE), the authors declare no financial support given or having a relationship with any organization may benefit from this article.

Authors' contributions: study conception, $A A$ and $G B$; review and editing, $A A, G B$, and $A K$; data collection and extraction, $A S$; supervision, $A K$, and GB; treating medical team; AK.

Acknowledgement: author would like to thanks to Dr. Nail Alward for participating in patient's management and care and Dr. Deyaa Mukhtar - head department of internal medicine- for his encouragement and supportive attitude.

\section{References}

1. Armin S, Wakil A, Tarbox J, Iwuji K.: COVID-19 vaccination: an attempt to control the pandemic. The Southwest Respiratory and Critical Care Chronicles. 2021, 9:32-

\section{9. $10.12746 /$ swrccc.v9i37.801}


2. Top 10 Companies Leading the Race of COVID-19 Vaccine. (2021). Accessed: 21 August 2021: https://intellizence.com/insights/top-10-companies-leading-the-race-of-covid-19vaccine/.

3. Interim recommendations for use of the Pfizer-BioNTech COVID-19 vaccine, BNT162b2, under Emergency Use Listing. (2021). Accessed: 21 August

2021: https://www.who.int/publications/i/item/WHO-2019-nCoV-vaccines-

SAGE recommendation-BNT162b2-2021.1.

4. Román GC, Gracia F, Torres A, Palacios A, Gracia K, Harris D: Acute transverse myelitis (ATM): clinical review of 43 patients with COVID-19-associated ATM and 3 post-vaccination ATM serious adverse events with the ChAdOx1 nCoV-19 vaccine (AZD1222). Front Immunol. 2021, 12:653786. 10.3389/fimmu.2021.653786

5. Sarma D, Bilello LA: A case report of acute transverse myelitis following novel coronavirus infection. Clin Pract Cases Emerg Med. 2020, 4:321-3. 10.5811/cpcem.2020.5.47937

6. Baghbanian SM, Namazi F: Post COVID-19 longitudinally extensive transverse myelitis (LETM)-a case report. Acta Neurol Belg. 2020, 10.1007/s13760-020-01497-x

7. Chow CC, Magnussen J, Ip J, Su Y: Acute transverse myelitis in COVID-19 infection. BMJ Case Rep. 2020, 13:10.1136/bcr-2020-236720

8. Zachariadis A, Tulbu A, Strambo D, Dumoulin A, Di Virgilio G: Transverse myelitis related to COVID-19 infection. J Neurol. 2020, 267:3459-61. 10.1007/s00415-020-09997-9

9. Kaur H, Mason JA, Bajracharya M, et al.: Transverse myelitis in a child with COVID-19. Pediatr Neurol. 2020, 112:5-6. 10.1016/i.pediatrneurol.2020.07.017

10. Valiuddin H, Skwirsk B, Paz-Arabo P: Acute transverse myelitis associated with SARS-CoV-2: a case-report. Brain Behav Immun Health. 2020, 5:100091. 10.1016/i.bbih.2020.100091

11. Munz M, Wessendorf S, Koretsis G, et al.: Acute transverse myelitis after COVID-19 pneumonia. J Neurol. 2020, 267:2196-7. 10.1007/s00415-020-09934-w

12. Hasan T, Khan M, Khan F, Hamza G: Case of Guillain-Barré syndrome following COVID-19 vaccine. BMJ Case Rep. 2021, 14:e243629. 10.1136/bcr-2021-243629

13. Selected Adverse Events Reported after COVID-19 Vaccination. (2021). Accessed: 21 August 2021: https://www.cdc.gov/coronavirus/2019-ncov/vaccines/safety/adverse-events.html.

14. AHA News: Experts Remain Confident About Vaccine Safety Monitoring. (2021). Accessed: 21 August 2021: https://consumer.healthday.com/aha-news-experts-remain-confidentabout-vaccine-safety-monitoring-2652793706.html.

15. Radwi M, Farsi S: A case report of acquired hemophilia following COVID-19 vaccine. J Thromb Haemost. 2021, 19:1515-8. 10.1111/ith.15291 
16. Reddy S, Reddy S, Arora M: A case of postural orthostatic tachycardia syndrome secondary to the messenger RNA COVID-19 vaccine. Cureus. 2021, 13:e14837. 10.7759/cureus.14837

17. Guo F, Zhang YB: Clinical features and prognosis of patients with Guillain-Barré and acute transverse myelitis overlap syndrome. Clin Neurol Neurosurg. 2019, 181:12732. 10.1016/j.clineuro.2019.04.014

18. Tahir N, Koorapati G, Prasad S, Jeelani HM, Sherchan R, Shrestha J, Shayuk M: SARS-CoV-2 vaccination-induced transverse myelitis. Cureus. 2021, 13:e16624. 10.7759/cureus.16624

19. Waheed W, Carey ME, Tandan SR, Tandan R: Post COVID-19 vaccine small fiber neuropathy. Muscle Nerve. 2021, 64:E1-2. 10.1002/mus.27251

20. Wise J: Covid-19: European countries suspend use of Oxford-AstraZeneca vaccine after reports of blood clots. BMJ. 2021, 372:n699. 10.1136/bmj.n699

21. Alimehmeti I: Efficacy and safety of AZD1222, BNT162b2 and mRNA-1273 vaccines against SARS-CoV-2. Albanian Journal of Trauma and Emergency Surgery. 2021, 5:7916. 10.32391/ajtes.v5i1.178

22. Finsterer J, Scorza FA: SARS-CoV-2 vaccines are not free of neurological side effects. Acta Neurol Scand. 2021, 144:109-10. 10.1111/ane.13451

23. Pagenkopf $C$, Südmeyer M: A case of longitudinally extensive transverse myelitis following vaccination against Covid-19. J Neuroimmunol. 2021, 358:577606. 10.1016/i.jneuroim.2021.577606

24. Singh Malhotra H, Gupta P, Prabhu V, Garg RK, Dandu H, Agarwal V: COVID-19 vaccinationassociated myelitis. QJM. 2021, 10.1093/aimed/hcab069

25. Fitzsimmons W, Nance CS: Sudden onset of myelitis after COVID-19 vaccination: an underrecognized severe rare adverse event. SSRN. 2021, 10.2139/ssrn.3841558

26. Baxter R, Lewis E, Goddard K, et al.: Acute demyelinating events following vaccines: a casecentered analysis. Clin Infect Dis. 2016, 63:1456-62. 10.1093/cid/ciw607 